$10-9-2015$

\title{
Healthcare Student Immunizations: An Overview
}

Douglas Gardenhire

Georgia State University, dgardenhire@gsu.edu

Follow this and additional works at: https://nsuworks.nova.edu/ijahsp

Part of the Health and Physical Education Commons, and the Medicine and Health Sciences Commons

\section{Recommended Citation}

Gardenhire D. Healthcare Student Immunizations: An Overview. The Internet Journal of Allied Health Sciences and Practice. 2015 Oct 09;13(4), Article 4.

This Manuscript is brought to you for free and open access by the College of Health Care Sciences at NSUWorks. It has been accepted for inclusion in Internet Journal of Allied Health Sciences and Practice by an authorized editor of NSUWorks. For more information, please contact nsuworks@nova.edu. 


\title{
Healthcare Student Immunizations: An Overview
}

\begin{abstract}
All clinical healthcare programs (CHP) in the United States require documentation of many types of immunizations and health information. The key for CHP personnel is to determine if immunization and health information is valid or if immunity exists. Documentation alone does not guarantee a student will be protected. This document will review common immunization and health information collected by many $\mathrm{CHP}$ and provide recommendations that programs may consider when adopting or changing polices on student immunization and health information.
\end{abstract}

\section{Author Bio(s)}

Douglas Gardenhire, EdD, RRT-NPS, FAARC, is interim chair of the Department of Respiratory Therapy at Georgia State University. 


\title{
IIJAHSP
}

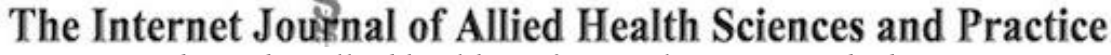

Dedicated to allied health professional practice and education

Vol. 13 No. 4 ISSN 1540-580X

\section{Healthcare Student Immunizations: An Overview}

\author{
Douglas Gardenhire, EdD, RRT. \\ Georgia State University \\ United States
}

\section{INTRODUCTION}

The collection of immunization records in the United State (U.S.) starts for most individuals no later than kindergarten. All states and U.S. territories have enacted laws governing immunizations to protect the welfare of society. ${ }^{1}$ Many states have exemptions based on medical, religious, and philosophical aims given proper documentation. ${ }^{2}$ Because of variations of law and exemptions, CHPs may find student's health records convoluted.

Immunization history and the health of students play an integral role in all CHPs placing students at clinical sites. CHPs often require different health and immunization documentation than state requirements. This paper looks at the issues associated with student health and immunization records and how it impacts the student and the school with recommendations CHPs may use for clinical placement.

\section{HEALTH REQUIRMENTS}

The most commonly required immunizations for CHP students may include documentation of measles, mumps, and rubella (MMR), a three part series for hepatitis B, varicella, tetanus/diphtheria acellular pertussis (Tdap), a tuberculosis (TB) skin test and influenza. Details for each will be discussed below.

\section{Measles, Mumps, and Rubella (MMR)}

The Edmonston measles vaccine was licensed in 1963. Following successful use of the vaccine, the mumps vaccine was licensed in 1967 while rubella (German measles) followed in 1969. As a result of the success of these individual vaccines, the combination of all three, measles, mumps, and rubella (MMR), was made available in 1971. ${ }^{3}$ The MMR vaccine is administered by a subcutaneous injection within the first year of life. One dose provides immunity for most individuals receiving the vaccination; however, a small number of patients do not develop immunity after receiving the first dose, so a second is routinely administered at 4-6 years of age to ensure the vaccine's total efficacy. The Centers for Disease Control (CDC) recommends two doses of MMR. 1

CHPs placing students into clinical rotations should require documented evidence through a serology report showing immunity for measles, mumps, and rubella. The CDC recommends a healthcare worker (HCW) born before 1957 that cannot not provide evidence of immunity to have two equally spaced doses of MMR. The two doses can be given as close as one month apart.4,5 However, for a HCW born after 1957, the CDC recommends those with documented evidence of two shots would suffice for immunity, even if serology determines negative or equivocal results.., 5

The overall incidence of these diseases has resulted in a perception of low risk; however, the current measles outbreak, which is attributed to Disney Land, California, continues to produce new cases regularly across the U.S. Another outbreak of measles occurred in Indiana in 2011, which was the largest outbreak since 1997. ${ }^{6}$ CHPs should consider having all students provide a serology report for measles, mumps, and rubella. If negative or equivocal results are reported it may be wise to consider a single dose of MMR before clinical placement. 


\section{Hepatitis B}

Hepatitis B virus is an infection that targets the liver. It is spread by contact with infected blood and body fluids. Acute infectious symptoms may include fever, nausea, vomiting, muscle aches, and jaundice. Chronic infection can lead to cirrhosis of the liver. ${ }^{7}$

The hepatitis B antigen was discovered by Baruch Blumberg in 1966. Irving Millman developed a blood test to screen for hepatitis B that has been in use since 1971. It was not until 1981 that a plasma-based vaccine was available. In 1986, the vaccine was synthetically engineered to contain no human products. ${ }^{7}$ In 1991, the increasing outbreak of human immunodeficiency virus (HIV) and reluctance of adult populations at risk to be vaccinated for hepatitis $B$ resulted in a recommendation by the CDC to immunize newborns. ${ }^{1}$

Hepatitis B can be spread by blood and body fluids. Since a HCW may be exposed, it is recommended they have documented evidence and immunity to hepatitis B. If a HCW cannot provide evidence, a three dose hepatitis B series should be given with dose one given immediately, dose two given one month later and the final dose given 5 months after the second. The individual should have immunity confirmed one to two months after the series has been completed. ${ }^{4,5} \mathrm{CHPs}$ should treat students in a similar fashion; however, immunity should be confirmed before clinical placement to determine if additional vaccination is required.

\section{Varicella}

Chickenpox is an airborne virus caused by the varicella zoster virus. The virus produces a blister-containing rash on the body and can also be found in the mouth. It is spread easily by coughing, sneezing, or direct contact with fluid from broken skin lesions (blisters). Symptoms include low-grade fever, nausea, and muscle aches, followed by the presence of the rash on the skin. ${ }^{8}$

Michiaki Takahashi developed the first varicella vaccine in 1974. It was not until 1995 that the U.S. had an approved varicella vaccine for use. ${ }^{8}$ Currently, the varicella vaccine is given in a two-dose series. The series is usually given during the first year of life and then again before entering elementary school. In 2005 the measles, mumps, rubella, and varicella (MMRV) vaccine was licensed in the U.S. ${ }^{1}$

A HCW should provide evidence of immunity by a 2-dose vaccination for varicella, serology, or documented healthcare practitioner diagnosis of varicella. If this is not done, a 2-dose vaccination, four weeks apart, is recommended. $4,5 \mathrm{CHPs}$ should require students provide evidence of immunity by serology to determine immunity before placement in clinical rotations. Documented disease and/or vaccination do not guarantee a student is immune.

\section{Tetanus, Diphtheria and Pertussis}

Tetanus is a disease of the nervous system caused by the bacterium Clostridium tetani. C. tetani produces two exotoxins, tetanolysis and tetanospasmin. Tetanospasmin is the neurotoxin that causes symptoms of tetanus. The most common early symptom is lockjaw -- muscle spasms of the jaw. Other symptoms include muscle spasms and stiffness throughout the body, difficulty swallowing, and seizure like activity. ${ }^{3}$ Tetanus is spread by cut or puncture of the skin. For example, a rusty nail or knife blade is quite common. However, it can be found in soil, and individuals may become infected through open wounds exposed to soil. A person infected with tetanus will not build immunity to future exposure. Vaccination is the only prevention method. Developed in 1924 by P. Descombey, the tetanus vaccine can be given individually or is commonly combined with diphtheria and pertussis. $^{9}$

Corynebacterium diphtheriae is a bacterium that causes upper airway swelling in patients. Other symptoms may include fever, sore throat and difficulty swallowing and breathing. Diphtheria can spread by direct contact or respiratory droplets. ${ }^{3,9}$ Emil von Behring is credited with the development of a vaccine for diphtheria in 1890 and Gaston Ramon created a heat inactivated diphtheria toxoid in 1923.

Pertussis, also known as whooping cough, is caused by the bacterium Bordetella pertussis. Pertussis can be spread by direct contact or respiratory droplets. ${ }^{3}$ It produces a harsh cough creating a "whooping" sound. Patients may also experience fainting from rapid breathing or vomiting from harsh coughing.

A pertussis vaccine had been created in the early 1900s. However, Thorvald Madsen is best known for creating a whole cell vaccine that was widely used in $1925 .{ }^{3}$ Since 1991, diphtheria, tetanus, and pertussis (DTaP) has been used to vaccinate children. The DTaP vaccine does not use the whole cell; rather, small purified pieces of the germ are used, and the vaccine is given 5 times from birth through 6 years of age. ${ }^{1}$ Tetanus, diphtheria, and pertussis (Tdap) contains a reduced diphtheria amount and is given as a booster to children and adolescents about every 10 years. ${ }^{1} \mathrm{HCWs}$ that do not have a well-documented vaccine

(c) The Internet Journal of Allied Health Sciences and Practice, 2015 
history should receive a one-time Tdap and a booster of tetanus and diphtheria ever 10 years. ${ }^{4,5}$ However, because pertussis has been on the rise around the United States, CHPs should affirm a student has been vaccinated against pertussis; otherwise, require a Tdap before clinical placement.

\section{Tuberculosis}

Tuberculosis (TB) is a bacterium caused by Mycobacterium tuberculosis. Robert Koch discovered it in 1882 . TB is not necessarily easy to diagnosis as many of the symptoms could be linked to other diseases. However, common symptoms include fever, cough, hemoptysis, fatigue, chest pain, and night sweats. TB is transmitted by respiratory droplets. ${ }^{10}$

Many patients that are infected with M. tuberculosis have a dormant form of TB termed latent. Individuals with latent tuberculosis infection (LTBI) have no signs or symptoms and cannot transmit the disease. LTBI can change to an active TB infection where the disease can spread. Because of this possibility, HCWs should be routinely screened for TB. ${ }^{10}$

The Mantoux tuberculin skin test, also known as purified protein derivative (PPD) or simply tuberculin skin test (TST), is the most widely used test to screen for TB. The test is named for Charles Mantoux who used the work of others to develop the test in 1907. The culture of TB is placed intradermal usually on the inside of the forearm and is read in 48-72 hours. The induration or reaction to the culture will determine possible exposure to TB by expanding in size from the original inoculation site and would harden. Individuals at low risk such as most HCWs would be negative if the induration was smaller than 15 millimeters (mm) in size. High risk groups such as those with compromised immune systems (i.e. HIV, organ transplants) would be positive if above $5 \mathrm{~mm}$ of induration. ${ }^{10,11}$ ( We actually use a two-step TB test which is becoming more common) https://www.ccsf.edu/en/studentservices/student-health-services/medical-service/tb-testing/_jcr_content/rightlinks/documentlink_0/file.res/Two-

Step\%20TB\%20Skin\%20Test.pdf

The use of a TST may not always be accurate and may give a false positive. This is commonly seen in individuals that have received the Bacillus Calmette-Guérin (BCG) vaccine. The BCG vaccine is not routinely recommended in the U.S. but is common in other countries, especially those with a higher incidence for TB. The BCG vaccine is an attenuated live bovine bacillus known as Mycobacterium bovis. The CDC recommends that individuals that have received the BCG vaccine with a positive TST be screened the same as those that have not had the BCG vaccine but have a positive TST. 10,11

A positive TST is indicative of further testing. To rule out active TB, a symptom survey and a chest X-ray (CXR) are completed. If a positive CXR and/or symptom survey leads to a diagnosis of active TB, then isolation and treatment with a combination antimycobacterials such as isoniazid (INH), rifampin (RIF), ethambutol (EMB), and/or pyrazinamide (PZA) is needed.10 However, a positive TST and a negative CXR should be considered LTBI. To further test, new whole blood testing utilizing interferongamma release assays (IGRAs) mix whole blood with antigens to detect proteins of $M$. tuberculosis. Two IGRAs that are utilized in the U.S. are QuantiFERON-TB Gold In-Tube test (QFT-GIT) and T-SPOT TB test (T-Spot). ${ }^{12}$ A negative result may assist to rule out LTBI. A positive result would trigger prophylactic treatment with monotherapy of INH or RIF or combination of INH and RIF. Prophylactic treatment is free of charge from county health departments across the U.S.

\section{Influenza}

The Centers for Disease and Control (CDC), the Advisory Committee on Immunization Practices (ACIP), and the Healthcare Infection Control Practices Advisory Committee (HICPAC) recommend that all U.S. health care workers become vaccinated annually against influenza. However, some individuals may have allergy concerns in taking the influenza vaccine. All currently available influenza vaccines are prepared by propagation of virus in embryonated chicken eggs with the exception of trivalent recombinant influenza vaccine FluBlok (Protein Sciences) and cell culture-based inactivated influenza vaccine Flucelvax (Novartis). A recent study of individuals with a history of severe allergic reaction to egg showed no anaphylaxis events, although some milder reactions were noted, suggesting that severe allergic reactions to egg-based influenza vaccines are not likely. ${ }^{13}$

\section{RECOMMENDATIONS AND CONCLUSIONS}

CHPs must be vigilant and protect the health of students as well as the health of those in clinical practice. This can be achieved by having a policy and procedure for immunizations and screening. Many of our students are young enough to have had most if not all of the previously discussed immunizations done as children. However, immunity can fade with time and may make students susceptible to infection. CHP should consider requiring students to provide serology over documented immunization for measles, mumps, rubella, hepatitis B, and varicella. Serology provides objective results compared to subjective history of vaccination or disease.

(C) The Internet Journal of Allied Health Sciences and Practice, 2015 
CHPs should require students to undergo a yearly TST while enrolled in their program. Students who have had the BCG vaccine should be treated the same as those with a positive TST. A positive TST should be followed by a negative CXR and prophylactic LTBI therapy before clinical placement. However, if students or the program feel strongly against prophylactic therapy, one of the two IGRAs could be considered to rule out TB infection.

CHP should consult their medical director and determine requirements from all clinical sites when developing a policy and procedure. However, going above and beyond minimum requirements may better protect students, patients, and the program.

\section{REFERENCES}

1. Centers for Disease Control. Vaccination Coverage and Surveillance. http://www.cdc.gov/VACCINEs/statssurv/schoolsurv/default.htm Accessed May 31, 2015.

2. National Vaccine Information Center. http://www.nvic.org/Vaccine-Laws/state-vaccine-requirements.aspx Accessed January $14,2015$.

3. van Panhuis WG1, Grefenstette J, Jung SY, Chok NS, Cross A, Eng H, Lee BY, Zadorozhny V, Brown S, Cummings D, Burke DS. Contagious diseases in the United States from 1888 to the present. N Engl J Med. 2013 Nov 28;369(22):2152-8.

4. Centers for Disease Control. Recommended Vaccines for Healthcare Workers. http://www.cdc.gov/vaccines/adults/recvac/hcw.html Accessed May 31, 2015.

5. Immunization of HCW: Recommendations of ACIP. MMWR. 2011;60(RR07):1-45.

6. CDC. Notes from the field: measles outbreak---Hennepin County, Minnesota, February--March 2011. MMWR 2011:60:421.

7. Beasley R. Development of Hepatitis B Vaccine. JAMA. 2009;302(3):322-324

8. Galea SA, Sweet A, Beninger P, Steinberg SP, Larussa PS, Gershon AA, Sharrar RG. The safety profile of varicella vaccine: a 10-year review. J Infect Dis. 2008 Mar 1;197 Suppl 2:S165-9.

9. Centers for Disease Control and Prevention. Epidemiology and Prevention of Vaccine-Preventable Diseases. Tetanus. Atkinson W, Wolfe S, Hamborsky J, Mclntyre L, eds. 11th ed. Washington DC: Public Health Foundation, 2009.

10. Centers for Disease Control. Tuberculosis. http://www.cdc.gov/tb/ Accessed May 31, 2015.

11. Daniel TM. The history of tuberculosis. Respir Med. 2006 Nov; 100(11):1862-70.

12. Updated Guidelines for Using Interferon Gamma Release Assays to Detect Mycobacterium tuberculosis Infection United States. MMWR 2010; 59 (RR-5); 1-25.

13. Des Roches A, Paradis L, Gagnon R, et al. Egg-allergic patients can be safely vaccinated against influenza. J Allergy Clin Immunol 2012;130:1213-6.e1. 